\title{
Terahertz characterisation of UV offset lithographically printed electronic-ink
}

\section{Article}

Accepted Version

Creative Commons: Attribution-Noncommercial-No Derivative Works 4.0

Zeng, Y., Edwards, M., Stevens, R., Bowen, J. W., Donnan, R. S. and Yang, B. (2017) Terahertz characterisation of UV offset lithographically printed electronic-ink. Organic Electronics, 48 (September). pp. 382-388. ISSN 1566-1199 doi:

https://doi.org/10.1016/j.orgel.2017.06.012 Available at https://centaur.reading.ac.uk/71085/

It is advisable to refer to the publisher's version if you intend to cite from the work. See Guidance on citing.

Published version at: https://doi.org/10.1016/j.orgel.2017.06.012

To link to this article DOI: http://dx.doi.org/10.1016/j.orgel.2017.06.012

Publisher: Elsevier

All outputs in CentAUR are protected by Intellectual Property Rights law, including copyright law. Copyright and IPR is retained by the creators or other copyright holders. Terms and conditions for use of this material are defined in the End User Agreement.

\section{www.reading.ac.uk/centaur}

\section{CentAUR}

Central Archive at the University of Reading 
Reading's research outputs online 


\title{
Terahertz Characterisation of UV Offset Lithographically Printed Electronic-Ink
}

\author{
Yang Zeng ${ }^{1,2}$, Marc Edwards ${ }^{3}$, Robert Stevens ${ }^{4}$, John Bowen ${ }^{5}$, Robert S Donnan ${ }^{1, *}$ and Bin Yang ${ }^{3, *}$ \\ ${ }^{1}$ School of Electronic and Computer Science, Queen Mary University of London, Mile End Road, London \\ E1 4NS, UK; ${ }^{2}$ College of Opto-electronic Science and Engineering, National University of Defense \\ Technology, Changsha, Hunan, 410073, China; ${ }^{3}$ Electronic and Electrical Engineering Department, \\ Thornton Science Park, University of Chester, CH2 4NU, UK; ${ }^{4}$ Nottingham Trent University, Burton \\ Street, Nottingham NG1 4BU, UK., ${ }^{5}$ Biomedical Engineering, School of Biological Sciences, University \\ of Reading, Whiteknights, Reading, RG6 6AY, UK.
}

\begin{abstract}
Inkjet-printed electronics are showing promising potential in practical applications, but methods for real-time, non-contact monitoring of printing quality are lacking. This work explores Terahertz (THz) sensing as an approach for such monitoring. It is demonstrated that alterations in the localised dielectric characteristics of inkjet-printed electronics can be qualitatively distinguished using quasi-optically-based, sub-THz reflection spectroscopy. Decreased reflection coefficients caused by the sintering process are observed and quantified. Using $\mathrm{THz}$ near-field scanning imaging, it is shown that sintering produces a more uniform spatial distribution of permittivity in the printed carbon patterns. Images generated using THz-TDS based imaging are presented, demonstrating the combination of high resolution imaging with quantification of complex permittivities. This work, for the first time, demonstrates the feasibility of quality control in printed electronic-ink with $\mathrm{THz}$ sensing, and is of practical significance to the development of in-situ and non-contact commercial-quality characterisation methods for inkjet-printed electronics.
\end{abstract}

Keywords: Inkjet-printed Electronics, THz sensing, spectroscopy, THz imaging

\section{Introduction}

Inkjet-printed electronics has been a popular topic of scientific research commensurate with advances in inkjet printing itself. The technology affords a number of advantages over conventional printing techniques, such as high edge definition, speed, printing onto flexible and fragile substrates, and low cost [1-3]. Its application widely covers the manufacture of organic thin-film transistors (OTFTs) [4, 5], organic light emitting devices (OLEDs) [6], radio frequency identification (RFID) tags [7, 8] and flexible solar cells [9], as well as fabrication of future plastic and polymer-based electronic devices [10-12].

Most inkjet printers operate on a drop-on-demand basis by spraying nano-particle-based dielectric or metallic inks onto the desired area to form the pattern. After the ink is dried, however, the constituent components of the ink, such as the stabiliser, prevent the nano-particles from binding. Hence, a sintering process is generally required to form an interconnection between the nano-particles in order to produce a high-quality dielectric/metallic layer [13, 14].

Obtaining uniform, continuous and high-quality printed features is key to achieve reliable electrical and electronic properties of the printed layers and consistent device performance. The quality of the print can be assessed using scanning electron microscopy (SEM). However, this method is relatively time consuming, as it requires a vacuum environment, and the imaging area is limited by the optics and electron beam. Additionally, SEM imaging mostly focuses on the surface profile of the printed layer instead of its intrinsic electrical properties [10]. Automated imaging (AI) technique, based on digital cameras to capture $2 \mathrm{D}$ images of dispersed particulate samples, is very powerful in providing geometric information (size and shape) of the particles [15], but again, cannot provide electrical properties. SEM and AI technologies therefore are not appropriate for real-time and large-area proofing of printed electronics. Alternatively, in industrial applications, sheet resistance is a more common measure of the quality of the printed ink pattern. Even so, sheet resistance measurement requires contact and is not coincident with the printing process $[16,17]$. 
However, electromagnetic remote sensing operating in the millimetre-wave and terahertz (THz) bands $(0.1-10 \mathrm{THz})$, has demonstrated a growing applicability to the disciplines of communications, materials, medical, chemical and life-sciences over the past two decades [18-21]. In addition to permitting noncontact probing, $\mathrm{THz}$ sensing affords non-ionising (hence non-destructive) probing by virtue of its low photon energies. These features make $\mathrm{THz}$ sensing a promising candidate for real-time quality control of large-area inkjet-printed products. Moreover, in contrast to circuit diagnostics using infrared thermography [22], THz sensing techniques probe the alteration in the dielectric properties of the samples under test, hence no active closed circuit is required. In this work, THz sensing of inkjet-printed electronics has been implemented in various modalities to investigate the capacity for assessing the print quality. The three modalities are: vector network analysis (VNA)-based sub-THz reflection spectroscopy; antenna near-field scanning ( $\mathrm{NSI}^{\mathrm{TM}}$ apparatus) and, THz time-domain spectroscopy (THz-TDS). The feasibility of each has been investigated on various printed ink patterns. The images and complex dielectric constants of the inks are used to reveal ink quality as a whole.

\section{Experimental}

\subsection{Sub-THz Quasi-Optical Reflection Spectroscopy}

$\mathrm{THz}$ reflection spectroscopy is performed by a free-space quasi-optical (QO) reflectometer. The reflectometer system is driven by a programmable network analyser (PNA, N5244A Keysight Tech) with a WR03 millimetre wave extension module (V03VNA2, OML Inc.), covering the frequency band 220 $325 \mathrm{GHz}$. The PNA measures the scattering parameters (S-parameters) to characterise the electrical behaviour of the network. In practice, directly measuring the reflectance from the samples under-test $\left(\mathrm{S}_{11}\right)$ with one port is problematic due to the interference of standing waves in the network and self-reflectance of the port. Instead, in our experimental configuration, a two-port network reflection set-up is implemented to characterise the dielectric properties of the samples under test. A schematic of the configuration of the reflectometer is illustrated in Fig. 1, including the QO path and the polarisation of the sub-THz wave.

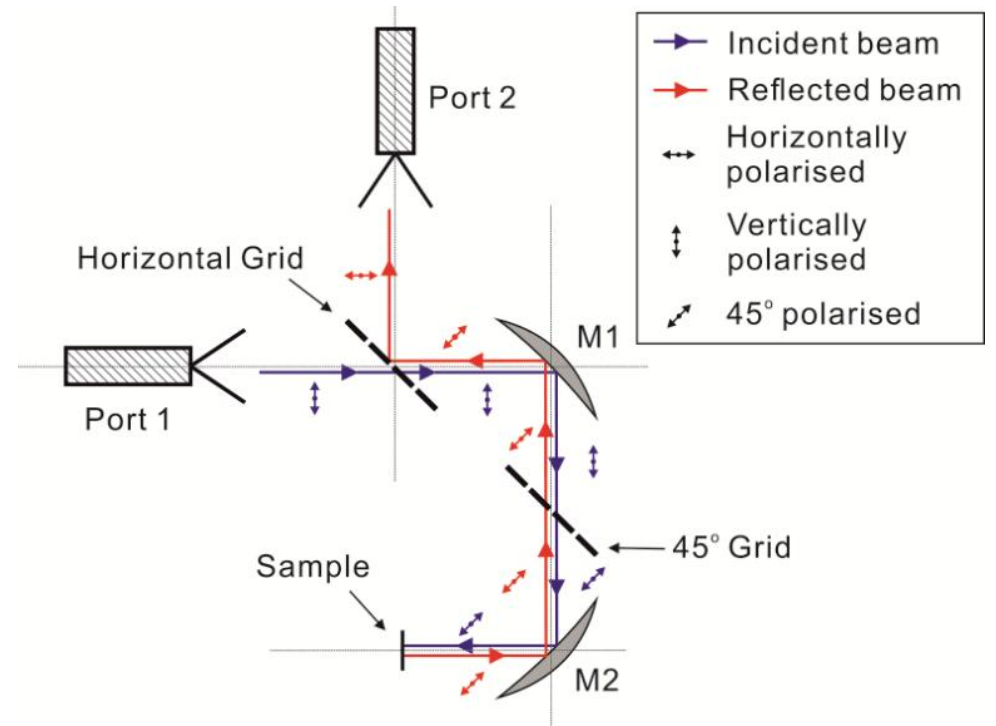

Figure 1: Schematic of the configuration of the sub-THz quasi-optical reflectometer. Blue and red lines represent the quasi-optical path of incident and reflected beams, respectively. The alteration in polarisation of the EM wave within the quasi-optical path is demonstrated with the polarisation indictors. 
The polarisation of the two ports are orthogonally pre-set in the measurement. The samples under-test are placed at the focus of the QO system, as indicated in the figure. With this QO configuration, the reflectance of the samples is represented by the transmittance from Port 1 to Port 2, i.e., $S_{21}$ parameter not conventional reflection coefficient $S_{11}$. Two wire grid polarisers with their wires at $0^{\circ}$ (horizontal) and $45^{\circ}$ to the plane of incidence, respectively, are used in combination with parabolic mirrors (M1 and M2) to transport the THz radiation through the QO network. The signal received by Port 2 is contributed by the reflection of the sample under test, the influence of the standing waves and self-reflectance in the circuit being suppressed.

In our study, the number of points in the frequency sweep is set to be 2001, with an intermediate frequency (IF) bandwidth of $50 \mathrm{kHz}$. This provides a spectral resolution of $52.5 \mathrm{MHz}$, whilst the scanning time for each data acquisition is about $2 \mathrm{~s}$. A calibration reference is established by measuring the reflectance from an optically-flat, polished, aluminium plate at the focus (i.e. the measurement position of the sample). The reference is re-checked for each measurement. The dynamic range of the system is better than $50 \mathrm{~dB}$ with the chosen parameters, and can be further improved by decreasing the IF bandwidth but at the expense of increased scan-time.

The spatial resolution of the measurement is fixed by the waist of the signal beam at the focus. When the system is well-aligned, the full waist of the probe-beam is smaller than $4 \mathrm{~mm}$. This is sufficiently less than the size of the printed pattern used in the measurements, and the QO reflectometer, therefore, provides localized electric properties of the samples under the test.

\subsection{THz Near-field Scanning Imager}

Similar to the THz QO reflectometer, the THz NSI system is driven by the same PNA (N5244A Keysight Tech. ) with a WR10 millimetre wave extension module (V10VNA2, OML Inc.) covering the frequency band from 95 to $105 \mathrm{GHz}$. The NSI system is used to scan the distribution of the printed surface through the measured reflectance of the samples. However, its modus operandi is different to the QO system. The configuration of the system is as shown in Fig. 2.

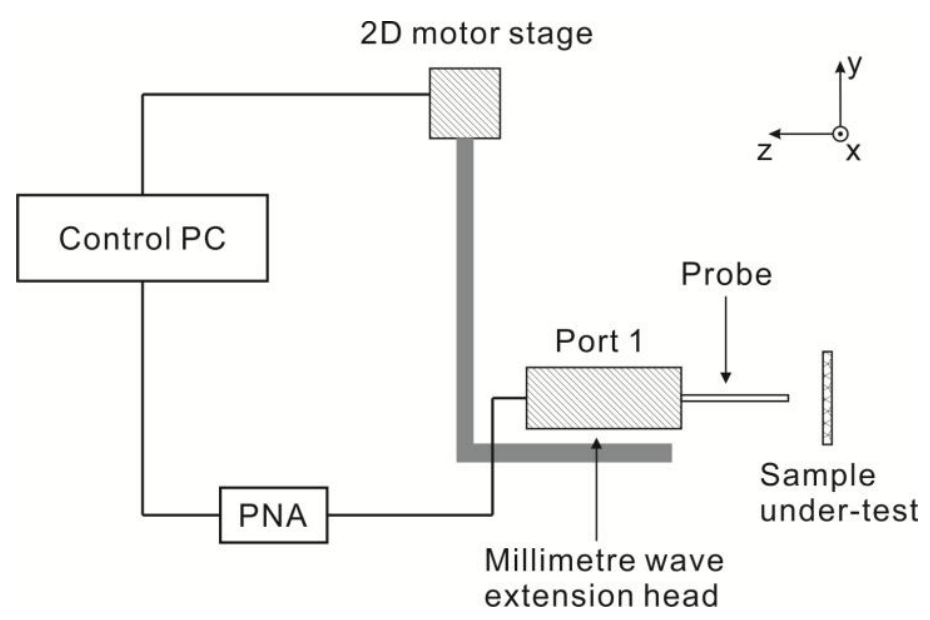

Figure 2: Configuration of the THz near-field scanning imaging system. The millimetre wave extension head is fixed to a 2D motor stage to scan in the $x y$ plane.

Instead of long-distance free-space sampling, the NSI system measures the near-field response of the sample under-test. The millimetre-wave extension head is fixed to a motorized near-field scanner, with the near-field probe tip connected to the VNA transceiver-port. During scanning, the probe tip is carried by the motorized scanner to build up a 2D scan (over an $x y$-plane) of the sample area. S-parameters are 
sampled to form the near-field image. The distance between the aperture of the probe tip and the sample is of the order of $3 \sim 5 \lambda$ ( $\lambda$ represented by the wavelength of band-centre of the extension head), in order to satisfy the near-field condition for imaging. The selected measurement band for imaging is $95-105$ $\mathrm{GHz}$, which is lower than for QO reflection spectroscopy. The reason is that higher frequencies, equating to shorter wavelengths, are more susceptible to phase-error via mechanical vibration suffered during measurement. Besides, reduced wavelength makes the system more sensitive to small distance deviation between the tip and the sample. This would create difficulties in the alignment of NSI 2D stage.

The spatial resolution of the scanning is set at $1 \mathrm{~mm}$, with the printed patterns fixed onto a polished quartz surface to eliminate effects of phase-shift due to ripples of the soft paper substrate. The distance between the probe tip and the samples is kept at $1 \mathrm{~cm}$ above the printed face to ensure that the phase-shift over the area is uniform. The imaging area is set to be a $3 \mathrm{~cm} \times 3 \mathrm{~cm}$ square in order to provide sufficient coverage of the printed circle area.

\subsection{THz Imaging based on Time-Domain Spectroscopy}

A standard THz time-domain spectroscopy system (THz-TDS, from TeTechs Ltd.) is used to measure the signal as a function of time-delay between the $\mathrm{THz}$ radiation and probe beam, in which the electric field of the $\mathrm{THz}$ pulse in the time-domain can be mapped. Fourier transforming the time-domain transmission data yields a spectral domain response workably spanning 0.2 to $3 \mathrm{THz}$ for a typical THz-TDS system. In combination with an $x y$ two-dimensional scanning stage, THz imaging can be realised to simultaneously map pattern quality and quantify the dielectric properties of the print. In this work, the scanning area is set as $20 \mathrm{~mm} \times 20 \mathrm{~mm}$ square with a spatial resolution of $0.5 \mathrm{~mm}$ in both $x$ - and $y$-directions to measure a meander-line pattern (total 1681 pixels). The scanning distance of the motor is set to be $5 \mathrm{~mm}$ with a 0.1 $\mathrm{mm} \cdot \mathrm{s}^{-1}$ step, so each point of the mapping takes approximately 50 seconds to capture a pixel with a spectral resolution of $14 \mathrm{GHz}$.

\section{Results and Discussion}

Using the sub-THz reflectometer, thirteen pairs of carbon inkjet $2 \mathrm{~cm}$ diameter circular samples were investigated. Each pair was printed onto a common substrate material with one of the pair sintered at 110 ${ }^{\circ} \mathrm{C}$ for one hour; the other being dried at room temperature. The measured and calculated reflection coefficient and permittivity of the samples are demonstrated in Fig. 3. The calculation is based on normalincidence reflection of the samples. For a normal-incidence wave, the reflection formula follows [23],

$$
r(\omega)=\frac{r_{1}-r_{1} \cdot e^{-\frac{i 2 \omega n(\omega) d}{c}}}{1-r_{1}^{2} \cdot e^{-\frac{i 2 \omega n(\omega) d}{c}}},
$$

where $\omega$ is the angular frequency of the incident wave; $r_{1}(\omega)$ is the single-surface reflectance; $n(\omega)=$ $\sqrt{\mu(\omega) \cdot \epsilon(\omega)}$ is the complex refractive index. In the reflection formula, the complex refractive index is determined by the complex permeability $\mu(\omega)$ and permittivity $\epsilon(\omega)$. For the case here, $\mu(\omega)=1$ as the samples have no magnetic properties; while $\epsilon(\omega)$ is further decomposed into its real part $\varepsilon_{1}(\omega)$ and the loss tangent $\tan \delta(\omega)$, i.e., $\varepsilon(\omega)=\varepsilon_{1}(\omega)+i \cdot \varepsilon_{1}(\omega) \cdot \tan \delta(\omega)$. The single-surface reflectance $r_{1}(\omega)$ is related to the reduced wave-impedance $Z$, which is correlated to the material's complex permeability and permittivity, i.e.,

$$
r_{1}(\omega)=\frac{Z-Z_{0}}{Z+Z_{0}}=\frac{\sqrt{\frac{\mu(\omega)}{\varepsilon(\omega)}-1}}{\sqrt{\frac{\mu(\omega)}{\varepsilon(\omega)}+1}}
$$


where $Z_{0}$ is wave impedance in free space $(120 \pi \Omega)$.

The measured complex reflection coefficients were fitted with Eq. (1) using a Levenberg-Marquardt square fitting procedure to calculate the complex permittivity of the samples. As the electronic ink samples have shown little dispersion in their dielectric properties, the acquired single pair of $\varepsilon_{1}(\omega)$ and $\tan \delta(\omega)$ is sufficient to interpret the character of the sample in the full measured spectrum (from 220 to $325 \mathrm{GHz})$.
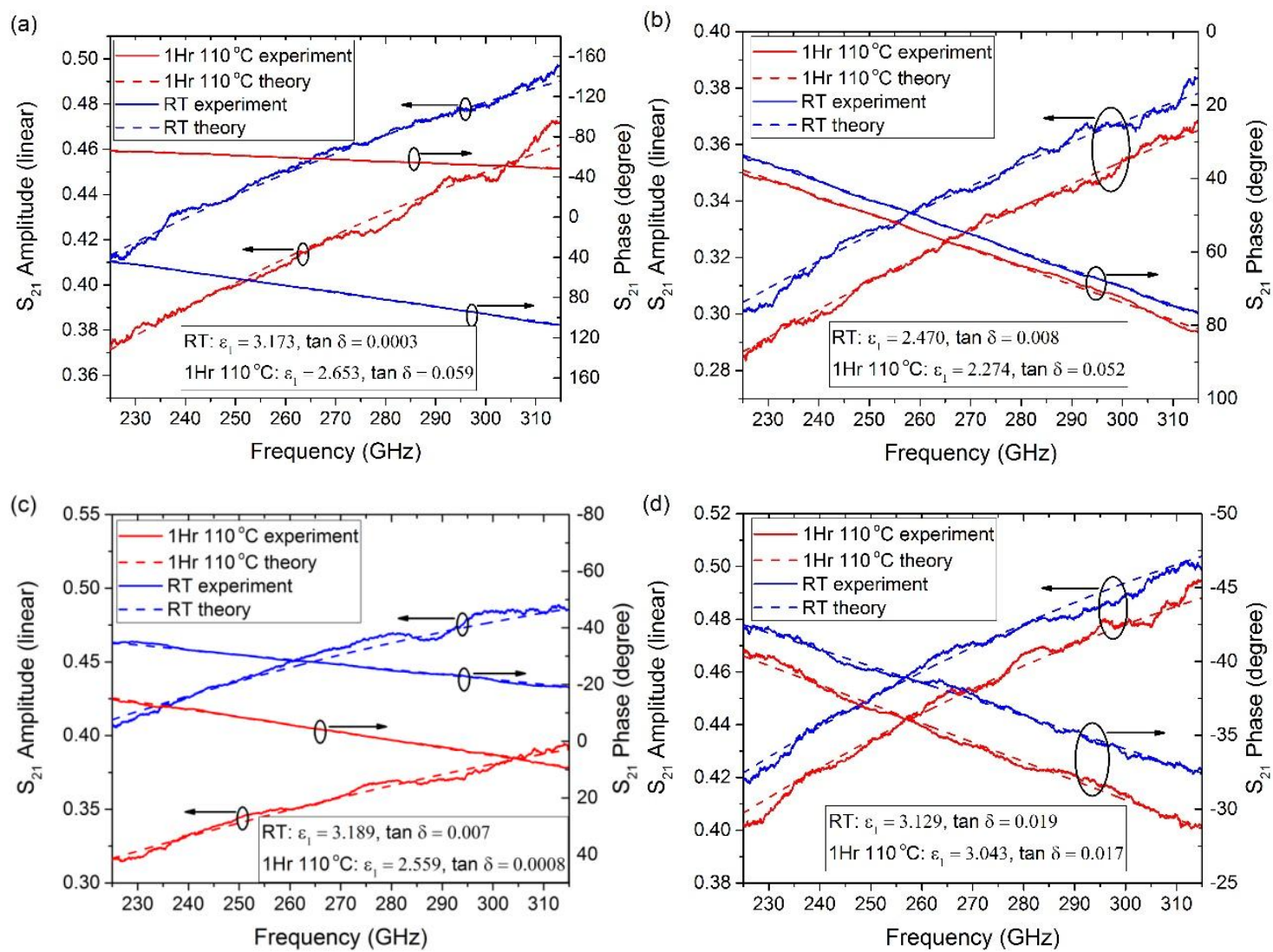

Figure 3: Comparison of measured and calculated reflection coefficient data from quasi-optical reflection spectroscopy in amplitude and phase of printed ink patterns on different paper substrates: (a) Choros 50; (b) Sora silk 65; (c) Alaketi; (d) Choros

Comparison is made for printed ink patterns on the same type of substrate paper. As shown in Fig. 3, sintered versus ambient-dried samples were easily distinguished by sub-THz reflectance spectra. Their difference is also marked in respective estimates of permittivity. It is noted that the real part of the permittivity is lower for the sintered samples. The decrement is between $2.3 \%$ to $19.8 \%$ over all groups. The decrement in the absolute value of $S_{21}$ corresponds to a weakened reflection of the sample. This can be attributed to the improved quality of the printed ink area after sintering (with the purging of residual moisture); i.e. sintering vaporises liquid residue within the ink, leading to increased conductivity.

Sub-THz reflection spectroscopy can provide local information of quality of the printed pattern. However, for practical commercial purposes, a global-area indication is needed for print-pattern quality. In order to observe the effect of the sintering process upon the distribution across a print of carbon-based ink, the samples were probed and imaged by the NSI system. The scanning parameters are set as discussed in Sec. 2.2. The near-field images of the two Choros samples corresponding to Fig. 3(d) at $100 \mathrm{GHz}$ are shown in Fig. 4. The imaging area was set slightly larger than the actual size of the round pattern (indicated by the 
dashed circles), to compensate for possible misalignment between the probe and the centre of the circle. The false-colour relative-amplitude distribution plots in Fig. 4 indicate that the distribution quality of the ink is better for the sintered sample. Sintering promotes a more uniform dielectric finish.

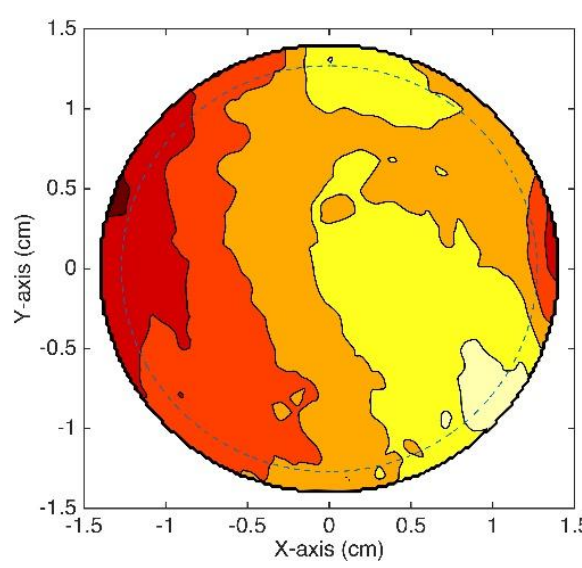

(a) Room temperature
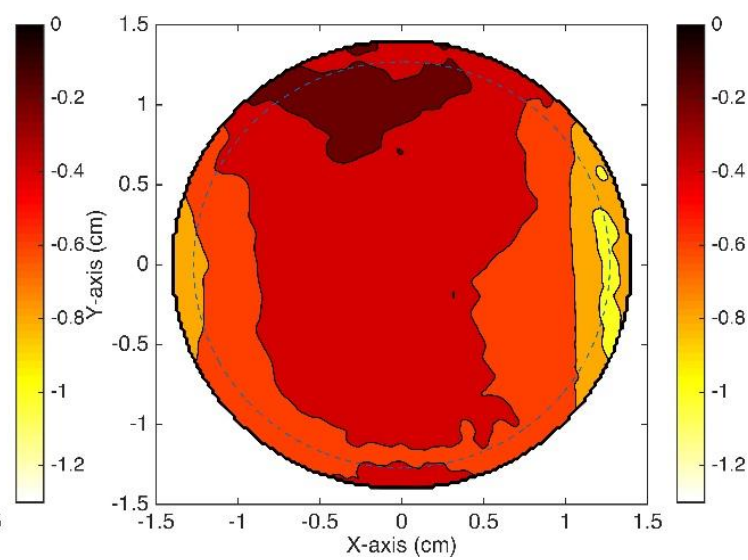

(b) 1 hour $110 \circ \mathrm{C}$

Figure 4: Relative false-colour amplitude near-field images of carbon-based ink prints on Choros paper at $100 \mathrm{GHz}$ (a) room temperature sample; (b) sample sintered at $110^{\circ} \mathrm{C}$ for 1 hour.

THz NSI additionally has the advantage of being able to distinguish samples printed on paper substrates having a high reflectance, such as silver paper. Such samples present a difficulty for sub-THz reflectance spectroscopy, where the variation in permittivity over the printed-area is obscured by the large reflectivity of the substrate, leading to a relatively large error when estimating the permittivity. This problem is overcome by NSI, as NSI focus on analysing the spatial information. The images at $100 \mathrm{GHz}$ of printed ink patterns on silver paper substrates, sintered at $110{ }^{\circ} \mathrm{C}$ for 1 hour and those free-drying at roomtemperature, are illustrated in Fig. 5.

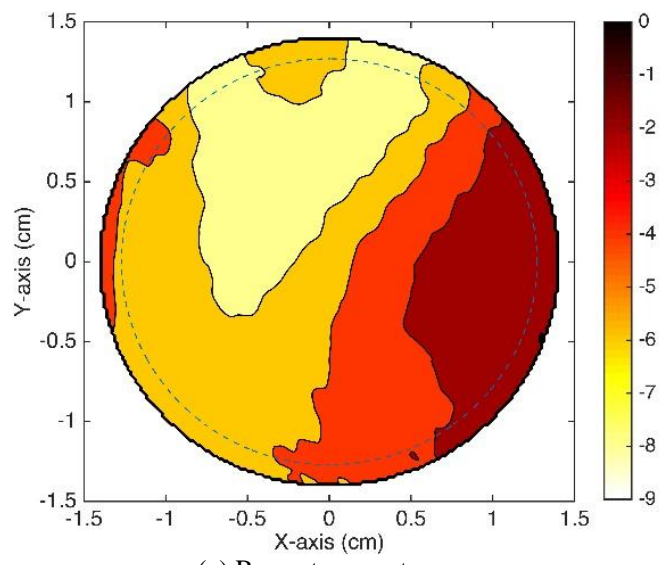

(a) Room temperature

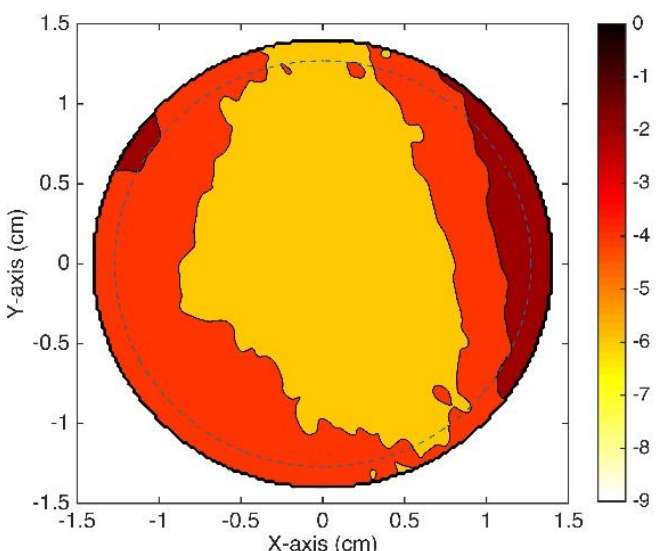

(b) 1 hour $110 \circ \mathrm{C}$

Figure 5: Relative false-colour amplitude near-field image of $S_{11}$ of printed ink on silver paper substrates at $100 \mathrm{GHz}$ (a) room temperature sample; (b) sample sintered at $110^{\circ} \mathrm{C}$ for 1 hour.

As illustrated in Fig. 5, although the amplitudes of $S_{11}$ in the two images are of similar scale, notable differences in the spatial distribution are apparent. A more uniformed distribution is observed for the sintered sample. This is consistent with the NSI images of patterns printed on Choros papers, and confirms the superior properties of the sintered samples. 
QO-based reflection spectroscopy and NSI have shown their merits in providing localised and overall information of the inkjet-printed patterns. THz-TDS-based imaging is considered as a good compromise technique that blends the advantages of QO-based reflection spectroscopy and NSI. It is capable of checking the quality of ink distribution whilst supplying local dielectric information. In this work, a 20 $\mathrm{mm} \times 20 \mathrm{~mm}$ square area of inkjet-printed meander-line is patterned, as shown in Fig. 6 (a) and is mapped by transmission THz-TDS as a demonstration. The width of the meander-line and white-space is $1.2 \mathrm{~mm}$; a $5 \mathrm{~mm}$-wide inkjet strip is located on the left-handed side and a $3 \mathrm{~mm}$-wide white-strip along the base. Although the minimum spot size of the $\mathrm{THz}$ beam is around $2 \mathrm{~mm}$ in diameter, the scanning steps at $0.5 \mathrm{~mm}$ intervals in both the $x$ - and $y$-axis are spatially fine enough to capture the $1.2 \mathrm{~mm}$ width of the meander-lines. The images at three different THz frequencies are presented in Fig. 6 (b-d).

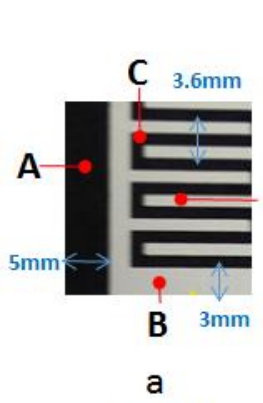

$0.7 \mathrm{THz}$

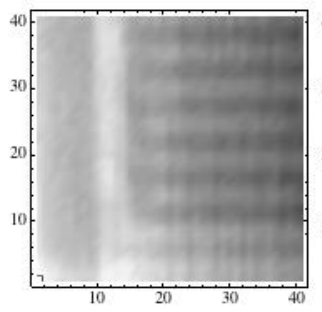

C

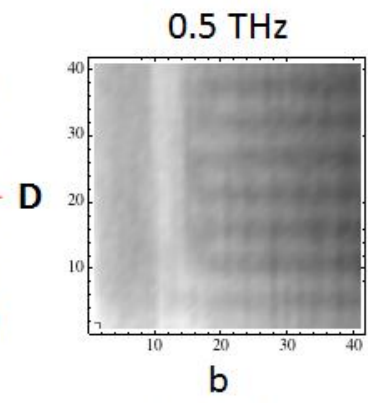

$1.2 \mathrm{THz}$

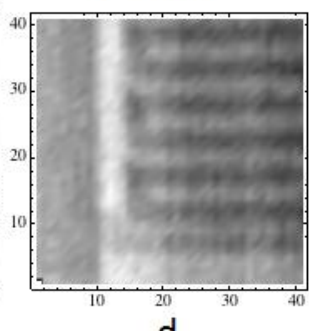

d
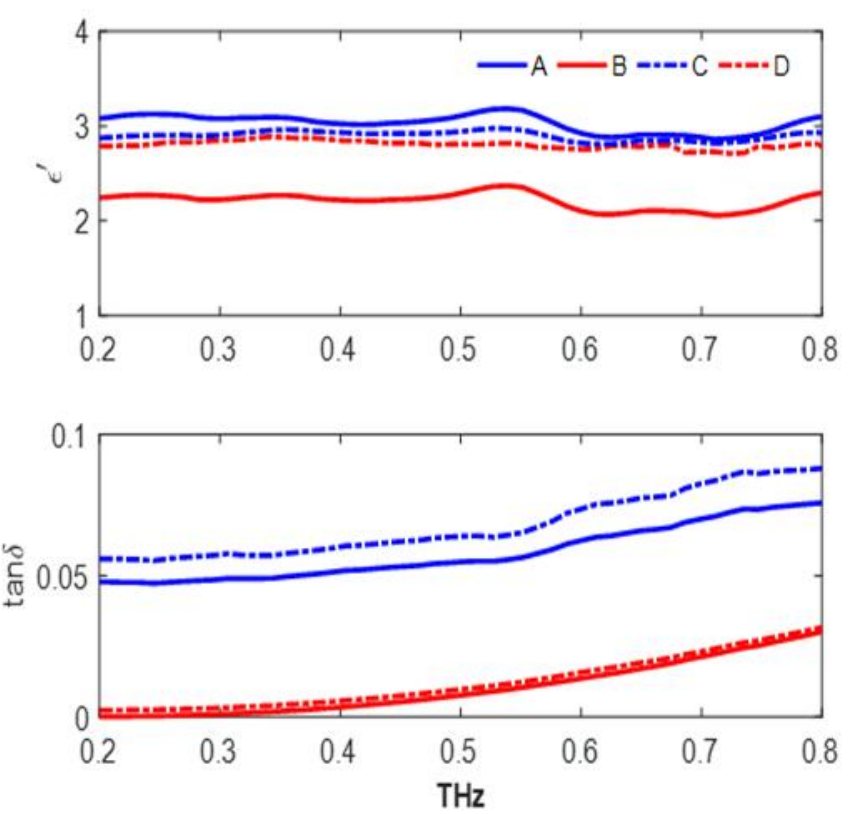

Figure 6: Transmission imaging measurement of a printed meander-line pattern by THz-TDS. Left: images at selected frequencies; Right: permittivities and loss tangents at selected points.

The image quality and resolution are enhanced, as expected, at the higher frequencies $(1.2 \mathrm{THz})$ of the $\mathrm{THz}$ band. However, realising these improvements is at the expense of a reduced system dynamic range. The time-domain spectroscopy results at four selected spots are used for the extraction of dielectric permittivities and loss tangents (shown in the right column plots in Fig. 6). To mimic the real inkjet-press conditions, measurements are undertaken at room temperature without pumping dry air into the space of the $\mathrm{THz}$ beam. The extracted parameters are considered to be valid between 0.2 and $0.8 \mathrm{THz}$ due to absence of strong water absorption lines there, nevertheless, a weak and irregular profile in both permittivities and loss tangents are observed around $0.56 \mathrm{THz}$, corresponding to a water line there. Dark inked carbon (point A) displays higher permittivity and loss compared to those of blank paper (point B). With the THz spot diameter of $2 \mathrm{~mm}$ (which is in excess of the width of the meander-line $(1.2 \mathrm{~mm})$ ), the data acquired at points $\mathrm{C}$ and $\mathrm{D}$ are effectively contributed to by both the black carbon ink and the blank paper substrate. At point $\mathrm{C}$, the black carbon ink makes a greater contribution, while the blank paper substrate gives a greater dielectric contribution to point $\mathrm{D}$. The permittivities at points $\mathrm{C}$ and $\mathrm{D}$ are lower than for point $\mathrm{A}$; however, they are higher than for the permittivity of blank paper. The loss at point $\mathrm{D}$ is slightly higher than that for the white paper. The loss tangent at point $\mathrm{C}$ is higher than that at the point $\mathrm{A}$, corresponding to the lower permittivity at the point $\mathrm{C}$. 
A comparison between the three $\mathrm{THz}$ analysis modalities implemented in this work for quality control of printed electronic-ink is summarised in Table 1. Although the specifications are related to the instruments used in our measurements, the comparison can still provide a generalised overview of the characteristics of the three techniques. Among them, sub-THz reflection spectroscopy offers the best spectroscopic resolution $(52.5 \mathrm{MHz})$. The data acquisition time per pixel is intermediate $(\sim 2 \mathrm{~s})$. For practical applications, this can be further reduced with compromise in the dynamic range. The system used in this work cannot provide information with regard to the spatial character of the printed electronic-ink, although this could be achieved with the assistance of motorised sample holders. The bandwidth of the system is limited by the $\mathrm{THz}$ extension heads and the cut-off frequency of the rectangular waveguide. This can be adjusted by changing the THz extension heads. The THz-TDS imaging system operates at the highest frequency with the broadest bandwidth, and provides the highest spatial resolution. Additionally, it can simultaneously provide dielectric and spatial information. However, it suffers from long scan times ( $\sim 50 \mathrm{~s}$ per pixel) and low spectral resolution $(14 \mathrm{GHz})$. With current developments in THz-TDS imaging systems, however, the scanning time can be significantly reduced to milliseconds with the implementation of vibrating mirrors or rotary optical delay lines (also with compromise in the dynamic range to 20 30 dB) [24]. Intermediate to sub-THz reflection spectroscopy and TDS is the NSI technique that maps spatial distribution at selected frequencies with faster scanning speed but lower spatial resolution. However, operating at selected frequencies, it cannot provide quantitative dielectric information due to the turbulence in phase over the measured area correlated to the ductile nature of the paper substrate.

Table 1: Comparison between sub-THz reflection spectroscopy, THz NSI and THz-TDS imaging techniques used in this work for

\begin{tabular}{|c|c|c|c|}
\hline & $\begin{array}{l}\text { Sub-THz reflection } \\
\text { spectroscopy }\end{array}$ & $\begin{array}{c}\text { THz Near-field scanning } \\
\text { imaging }\end{array}$ & $\begin{array}{c}\text { THz Time domain } \\
\text { spectroscopy imaging }\end{array}$ \\
\hline $\begin{array}{l}\text { Permittivity } \\
\text { measurement }\end{array}$ & Yes & No & Yes \\
\hline $\begin{array}{c}\text { Spatial } \\
\text { distribution } \\
\text { mapping }\end{array}$ & $\begin{array}{c}\text { No (capable with } \\
\text { motorised sample } \\
\text { holder) }\end{array}$ & Yes & Yes \\
\hline $\begin{array}{l}\text { Operation } \\
\text { frequency }\end{array}$ & $\begin{array}{c}220 \mathrm{GHz}-325 \mathrm{GHz} \\
\text { (adjustable by changing } \\
\text { extension heads [25]) }\end{array}$ & $\begin{array}{c}\text { Selected frequencies } \\
\text { between } 95-105 \mathrm{GHz}\end{array}$ & $0.2 \mathrm{THz}-3.5 \mathrm{THz}$ \\
\hline Dynamic range & $>55 \mathrm{~dB}$ & $>50 \mathrm{~dB}$ & $\begin{array}{l}\sim 60 \mathrm{~dB} @ 1.0 \mathrm{THz} \\
\sim 20 \mathrm{~dB} @ 3.0 \mathrm{THz}\end{array}$ \\
\hline Spectral resolution & $52.5 \mathrm{MHz}$ & $1 \mathrm{GHz}^{*}$ & $14 \mathrm{GHz}$ \\
\hline Spatial resolution & $\sim 2 \mathrm{~mm}$, not imaging & $\sim 1 \mathrm{~mm}$ & $\sim 0.5 \mathrm{~mm}$ \\
\hline $\begin{array}{l}\text { Scanning time per } \\
\text { pixel }\end{array}$ & $\sim 2 \mathrm{~s}$ & $\sim 0.5 \mathrm{~s}$ & $\begin{array}{l}\sim 50 \mathrm{~s} \text { (can be } \\
\text { significantly shortened } \\
\text { with different set-ups } \\
\text { and fast scan }\end{array}$ \\
\hline
\end{tabular}


\begin{tabular}{l|l|l|c}
\hline & & technologies [24]) \\
\hline
\end{tabular} frequencies.

In this work, the comparison of the inkjet-printed electronics is limited to patterns on the same substrate materials. The reason is that the response of the printed patterns to the THz wave is contributed to by both the printed graphite ink layer and the substrate paper. Permittivities extracted directly from the measurements represent not only the quality of the printed graphite layer, but also the electrical behaviour of the substrate material. Nevertheless, it is possible to extract the dielectric properties of the printed graphite layer itself, subjected to a priori information of the substrate paper in the QO reflectometry system [26], or by using advanced deconvolution de-embedding imaging algorithms in the THz-TDS scheme [27].

One drawback of $\mathrm{THz}$ sensing is the relatively poor spatial resolution (several wavelengths) due to the diffraction limit of the $\mathrm{THz}$ wave. This can be improved to within $1 \lambda$ by implementing beam-forming techniques [28], and further reduced by increasing the carrier frequency of the probing $\mathrm{THz}$ wave (with quantum cascade lasers [29]). Super-resolution techniques are also the option to reconstruct the images to improve the resolution [30]. Meanwhile, sub-wavelength detection and imaging techniques such as $\mathrm{THz}$ near-field probing [31] and $\mathrm{THz}$ plasmonic assisted imaging [32] are also under rapid development. Measuring with the evanescent waves, the spatial resolution can reach as high as $3 \mu \mathrm{m}$. These approaches show the prospect of high resolution probing of printed-electronics with $\mathrm{THz}$ sensing.

\section{Conclusion}

In this study, several novel methodologies, based on $\mathrm{THz}$ sensing, are introduced for monitoring the quality of inkjet-printed electronics; these are quasi-optic-based sub- $\mathrm{THz}$ reflection spectroscopy; $\mathrm{THz}$ near-field scanning imaging; and, $\mathrm{THz}$ time-domain spectroscopy based imaging. To the best of our knowledge, this paper reports the first measurement demonstration of non-contact quality-monitoring of inkjet-printed electronic devices based on $\mathrm{THz}$ sensing technologies. This is of significant commercial utility in the application of inkjet-printed electronics.

In our measurements, the variation in permittivity associated with post-annealing of the print, was characterised using QO-based sub-THz reflection spectroscopy. In the spectrum between 220 and 325 $\mathrm{GHz}$, printed electronic samples sintered at $110{ }^{\circ} \mathrm{C}$ for 1 hour return a lower reflection, corresponding to a lower permittivity. This correlates to the improved quality of the printed pattern resulting from the hightemperature post-processing. This trend is followed for all groups of printed patterns compared in our measurements, demonstrating the viability of quality-assurance via $\mathrm{THz}$ reflection spectroscopy. Furthermore, the influence of high-temperature sintering on the spatial distribution of the inkjet-printed electronic patterns was investigated by THz NSI. The sintered samples return a more even image of $S_{11}$ distribution, hence more uniform dielectric characteristics across the print-pattern. THz-TDS-based imaging is able to both map and characterise the printed electronic-inkjet samples. This approach introduces the possibility of simultaneously obtaining dielectric and spatial information of the samples. In conclusion, three common $\mathrm{THz}$ modalities have been compared, with the result that $\mathrm{THz}$ technology has been demonstrated to provide a means for process control of sheet-to-sheet and roll-to-roll printing in large-area electronics manufacturing. The focus of future work will be to speed up the data acquisition rate sufficiently for $\mathrm{THz}$ techniques to be usable on the production line.

\section{Acknowledgements}


Y. Z. gratefully acknowledges the financial support from China Scholarship Council (CSC); B.Y. gratefully acknowledges the financial support of the EPSRC Centre for Innovative Manufacturing in Large Area Electronics (EP/K03099X/1).

\section{References}

[1] H.W. Choi, T. Zhou, M. Singh, G.E. Jabbour, Recent developments and directions in printed nanomaterials, Nanoscale, 7 (2015) 3338-3355.

[2] M. Berggren, D. Nilsson, N.D. Robinson, Organic materials for printed electronics, Nat Mater, 6 (2007) 3-5.

[3] E. Sowade, M. Polomoshnov, R.R. Baumann, The design challenge in printing devices and circuits: Influence of the orientation of print patterns in inkjet-printed electronics, Organic Electronics, 37 (2016) 428-438.

[4] D.-Y. Shin, P.J. Smith, Theoretical investigation of the influence of nozzle diameter variation on the fabrication of thin film transistor liquid crystal display color filters, J Appl Phys, 103 (2008) 114905.

[5] E. Sowade, K.Y. Mitra, E. Ramon, C. Martinez-Domingo, F. Villani, F. Loffredo, H.L. Gomes, R.R. Baumann, Up-scaling of the manufacturing of all-inkjet-printed organic thin-film transistors: Device performance and manufacturing yield of transistor arrays, Organic Electronics, 30 (2016) 237-246.

[6] H.M. Haverinen, R.A. Myllylä, G.E. Jabbour, Inkjet printing of light emitting quantum dots, Applied Physics Letters, 94 (2009) 073108.

[7] S. Kim, C. Mariotti, F. Alimenti, P. Mezzanotte, A. Georgiadis, A. Collado, L. Roselli, M.M. Tentzeris, No Battery Required: Perpetual RFID-Enabled Wireless Sensors for Cognitive Intelligence Applications, IEEE Microwave Magazine, 14 (2013) 66-77.

[8] S. Molesa, D.R. Redinger, D.C. Huang, V. Subramanian, High-quality inkjet-printed multilevel interconnects and inductive components on plastic for ultra-low-cost RFID applications, Materials Research Society Symposium Proceedings, Warrendale, Pa.; Materials Research Society; 19992003, pp. 253-258.

[9] F.C. Krebs, Fabrication and processing of polymer solar cells: a review of printing and coating techniques, Sol Energ Mat Sol C, 93 (2009) 394-412.

[10] B.S. Cook, Y. Fang, S. Kim, T. Le, W.B. Goodwin, K.H. Sandhage, M.M. Tentzeris, Inkjet catalyst printing and electroless copper deposition for low-cost patterned microwave passive devices on paper, Electronic Materials Letters, 9 (2013) 669-676.

[11] M. Walther, A. Ortner, H. Meier, U. Löffelmann, P.J. Smith, J.G. Korvink, Terahertz metamaterials fabricated by inkjet printing, Applied Physics Letters, 95 (2009) 251107.

[12] R.E. Sousa, C.M. Costa, S. Lanceros-Méndez, Advances and Future Challenges in Printed Batteries, ChemSusChem, 8 (2015) 3539-3555.

[13] J. Perelaer, M. Klokkenburg, C.E. Hendriks, U.S. Schubert, Microwave Flash Sintering of InkjetPrinted Silver Tracks on Polymer Substrates, Advanced Materials, 21 (2009) 4830-4834.

[14] H.K. Seung, P. Heng, P.G. Costas, K.L. Christine, M.J.F. Jean, P. Dimos, All-inkjet-printed flexible electronics fabrication on a polymer substrate by low-temperature high-resolution selective laser sintering of metal nanoparticles, Nanotechnology, 18 (2007) 345202.

[15] S. Venkataraman, D.P. Allison, H. Qi, L.M. Jennifer, N.L.Kallewaard, J.E.Crowe, M.J Doktycz, Automated image analysis of atomic force microscopy images of rotavirus particles, Ultramicroscopy, 106 (2006): 829-837.

[16] M. Singh, H.M. Haverinen, P. Dhagat, G.E. Jabbour, Inkjet printing-process and its applications, Advanced materials, 22 (2010) 673-685. 
[17] J. Perelaer, P.J. Smith, D. Mager, D. Soltman, S.K. Volkman, V. Subramanian, J.G. Korvink, U.S. Schubert, Printed electronics: the challenges involved in printing devices, interconnects, and contacts based on inorganic materials, Journal of Materials Chemistry, 20 (2010) 8446-8453.

[18] J. Federici, L. Moeller, Review of terahertz and subterahertz wireless communications, J Appl Phys, 107 (2010) 111101.

[19] A.I. McIntosh, B. Yang, S.M. Goldup, M. Watkinson, R.S. Donnan, Terahertz spectroscopy: a powerful new tool for the chemical sciences?, Chemical Society Reviews, 41 (2012) 2072-2082.

[20] T. Yasui, Y. Kabetani, E. Saneyoshi, S. Yokoyama, T. Araki, Terahertz frequency comb by multifrequency-heterodyning photoconductive detection for high-accuracy, high-resolution terahertz spectroscopy, Applied Physics Letters, 88 (2006) -.

[21] M. Naftaly, R.E. Miles, Terahertz time-domain spectroscopy for material characterization, P Ieee, 95 (2007) 1658-1665.

[22] R. Heiderhoff, A. Makris, T. Riedl, Thermal microscopy of electronic materials, Materials Science in Semiconductor Processing, 43 (2016) 163-176.

[23] M. Born and E. Wolf, Principles of Optics, 6th ed. New York: Pergamon, 1980.

[24] G.-J. Kim, S.-G. Jeon, J.-I. Kim, Y.-S. Jin, High speed scanning of terahertz pulse by a rotary optical delay line, Rev Sci Instrum, 79 (2008) 106102.

[25] Y. Bin, R.J. Wylde, D.H. Martin, P. Goy, R.S. Donnan, S. Caroopen, Determination of the Gyrotropic Characteristics of Hexaferrite Ceramics From 75 to $600 \mathrm{GHz}$, Microwave Theory and Techniques, IEEE Transactions on, 58 (2010) 3587-3597.

[26] Y. Bin, S. Kastriot, L. Xiaoming, S. Hansheng, S.D. Robert, Complex Permittivity of Pure Water Measured by Vector Network Analysis at W-Band, Journal of Physics: Conference Series, 286 (2011) 012005 .

[27] G.C. Walker, J.W. Bowen, J. Labaune, J.-B. Jackson, S. Hadjiloucas, J. Roberts, G. Mourou, M. Menu, Terahertz deconvolution, Opt Express, 20 (2012) 27230-27241.

[28] M. Shalaby, C.P. Hauri, Demonstration of a low-frequency three-dimensional terahertz bullet with extreme brightness, Nat Commun, 6 (2015).

[29] A.W. Lee, B.S. Williams, S. Kumar, Q. Hu, J.L. Reno, Real-time imaging using a 4.3-THz quantum cascade laser and a $320 \times 240$ microbolometer focal-plane array, Ieee Photonic Tech L, 18 (2006) 14151417.

[30]Linwei Yue, Liangpei Zhang, Image super-resolution: The techniques, applications and future. Signal Processing, vol 128, nov 2016, page 389-408.

[31] A.J. Macfaden, J.L. Reno, I. Brener, O. Mitrofanov, $3 \mu \mathrm{m}$ aperture probes for near-field terahertz transmission microscopy, Applied Physics Letters, 104 (2014) 011110.

[32] S. Kawata, Y. Inouye, P. Verma, Plasmonics for near-field nano-imaging and superlensing, Nat Photon, 3 (2009) 388-394. 